\title{
Resource Allocation for Energy Efficiency in OFDMA-Enabled WPCN
}

\author{
Tien-Tung Nguyen, Quoc-Viet Pham, Van-Dinh Nguyen, Jong-Ho Lee, and Yong-Hwa Kim
}

\begin{abstract}
This letter considers a wireless powered communication network (WPCN), where an energy-constrained device directly uses harvested energy from a power transfer source to transmit independent signals to multiple Internet of Thing (IoT) users using orthogonal frequency division multiple access (OFDMA). Our goal is to maximize the system energy efficiency (EE) by jointly optimizing the duration of energy harvesting (EH), subcarrier and power allocation. The formulated problem is a mixed integer nonlinear programming (MINLP) problem due to the presence of binary assignment variables, and thus it is very challenging to solve it directly. By leveraging Dinkelbach method, a very efficient iterative algorithm with closed-form solutions in each iteration is developed, where its convergence is guaranteed. Numerical results show that the proposed algorithm obtains a fast convergence and outperforms baseline algorithms. Notably, they also reveal that the power source should transmit its maximum allowable power to obtain the optimal EE performance.
\end{abstract}

Index Terms-Energy-efficiency, IoT, OFDMA, subcarrier assignment, WPCN.

\section{INTRODUCTION}

C OMMUNICATION plays an important role in disaster situations where communication infrastructure and power disruptions result in low reliability and reduced availability of the network [1]. In many cases, communication networks in disaster areas are unavailable because of power failure. Equipments such as base stations and access points are not functional due to this loss of power. Further, Internet of Things (IoT) devices are typically with low-power and low-cost requirements with a finite-capacity battery, and the replacement is not always available. Therefore, a vital task during disasters is the restoration of power supplies.

To overcome this issue, energy harvesting $(\mathrm{EH})$ through renewable energy sources (e.g., wind, solar, geothermal, and hydropower) is advocated as a promising solution to prolong

Tien-Tung Nguyen is with the Department of Electronic Engineering, Myongji University, Yongin 17058, South Korea and Telecommunication Division, Industrial University of Ho Chi Minh City, Ho Chi Minh City 700000, Vietnam (email: nguyentientung@iuh.edu.vn).

Quoc-Viet Pham is with the Research Institute of Computer, Information and Communication, Pusan National University, Busan 46241, South Korea (email: vietpq@pusan.ac.kr).

Van-Dinh Nguyen is with the Interdisciplinary Centre for Security, Reliability and Trust (SnT), University of Luxembourg, L-1855 Luxembourg City, Luxembourg (email: dinh.nguyen@uni.lu).

Jong-Ho Lee is with the School of Electronic Engineering, Soongsil University, Seoul 06978, South Korea (email: jongho.lee@ssu.ac.kr).

Yong-Hwa Kim is with the Department of Electronic Engineering, Myongji University, Yongin 17058, South Korea (email: yongkim@mju.ac.kr).

This work was supported in part by Korea Electric Power Corporation (Grant number: R17XA05-22), and in part by the National Research Foundation of Korea (NRF) funded by Korea government (MSIT) (2019R1A2C2086621). The work of V.-D. Nguyen was supported in part by the Luxembourg National Research Fund (FNR) in the framework of the FNR-FNRS bilateral project "InWIP-NETs: Integrated Wireless Information and Power Networks." the life-time of IoT devices. However, in disaster situations, such energy sources may not be a viable approach due to destrustion. Therefore, wireless power transmission using radio frequency (RF) to charge for energy-constrained devices is a promising solution for low-power wireless networks [1]-[5]. In [4], a hybrid power transfer architecture was proposed where airships, helicopters, and balloons are deployed as power supply sources to transfer energy wirelessly via inductive power transfer to large communication devices and via microwave power transfer to small communication devices. However, the authors only focused on describing the proposed hybrid power transfer procedure without providing any mathematical analyses. The authors in [5] investigated the sum-rate of an IoT wireless powered communication network (WPCN), while the energy efficiency (EE) of the network was not considered.

Due to the explosive growth of high-data-rate applications and services, the energy consumption continues to increase dramatically. Moreover, faced with the rapid rise in energy costs, EE has been widely studied as a key performance indicator in both academia and industry [6]-[11]. In WPCNs, EE will undoubtedly be considered as a significant figure-ofmerit in the design of WPCN networks because the energy of a network is consumed in both wireless energy transfer (WET) and wireless information transfer (WIT) phases [6][10]. Moreover, focusing on throughput without regard to EE is not guaranteed to be practical for the lifespan of the network due to the greedy use of power [11]. This approach is not suitable for energy-constrained devices or the network, especially in disaster situations where the power sources may be disconnected. This EE problem is addressed in our letter.

In [6], a cyclic iteration method was proposed to maximize the system EE in a time division multiple access (TDMA)enabled WPCN. To improve the spectrum usage, the works in [7] and [8] considered the system EE maximization problem of time division mode switching (TDMS)-enabled WPCN and non-orthogonal multiple access (NOMA)-enabled WPCN, respectively. However, the transmit power at the access point (AP) was not jointly optimized in these works.

In this letter, we consider a WPCN where an energyconstrained AP deploys 'harvest-then-transmit' protocol [6][8] to harvest energy from an external power source for data transmission to multiple IoT devices using orthogonal frequency division multiple access (OFDMA). Unlike [5], our objective is to maximize the system EE by jointly optimizing EH time, subcarrier (SC) and power allocation at the AP. To obtain a better performance, in this work the transmit power and SC assignment at AP in the WIT phase are jointly optimized, which is essentially different from [6]-[8].

Aiming at the EE performance, we first introduce new binary variables to establish IoT user-SC associations, and then 
formulate the system EE maximization problem of OFDMAenabled WPCN. The problem design belongs to the difficult class of MINLP, which is troublesome to solve directly. We first determine the optimal EH time after justifying the peak power used at the power source to provide more insight into the structure of the solution. Next, an alternative algorithm based on the Dinkelbach method is provided to tackle the problem, which obtains good performance while taking advantages of fast convergence and low computational complexity. We note that the proposed iterative algorithm does not invoke any existing solvers [3], since the closed-form solutions for SC and power allocation are found at each iteration. Numerical results are given to demonstrate the fast convergence rate of the proposed algorithm, which is independent with the problem size (i.e., the number of antennas at the AP). They also reveal the improvement performance of the proposed algorithm over state-of-the-art approaches.

\section{SYSTEM MODEL AND POWER CONSUMPTION MODEL}

\section{A. System Model}

We consider a WPCN where a single-antenna power station (PS) transfers energy to an energy-constrained AP equipped with $N_{T}$ antennas for data transmission to $K$ single-antenna IoT users via OFDMA. The letter focuses on the OFDMAenabled WPCN due to the easy implementation and low complexity of the algorithms and hardware. In addition, OFDMA is standardized and used in narrowband-IoT networks [12]. A total transmission time block is denoted as $T_{\max }$. Without loss of generality, $T_{\max }$ is normalized to one, i.e., $T_{\max }=1$. The total transmission time block is divided into two phases consisting of the WET phase, denoted $\tau_{0}$, and the WIT phase, denoted $1-\tau_{0}$. Further, it is assumed that the perfect channel state information (CSI) is obtained at transceivers [5]. The sets of users and subcarriers are denoted by $\mathcal{K} \triangleq\{1, \cdots, K\}$ and $\mathcal{N} \triangleq\{1, \cdots, N\}$, respectively.

In OFDMA-enabled WPCN, the total system bandwidth $B$ is equally divided into $N$ orthogonal subcarriers (SCs) and the bandwidth for each $\mathrm{SC}$ is denoted by $W=B / N$. The channel vector from the PS to AP over the $n^{t h} \mathrm{SC}$ is denoted as $\mathbf{h}_{p, n} \in \mathbb{C}^{N_{T} \mathbf{x} 1}$. In the WET phase, the amount of harvested energy can be calculated as $E=\eta P_{0} \sum_{n \in \mathcal{N}}\left\|\mathbf{h}_{p, n}\right\|^{2} \tau_{0}$ where $P_{0}$ is the transmit power at the PS, and $0<\eta \leq 1$ is the energy conversion efficiency of the AP. For simplicity, the maximum ratio transmission (MRT) technique is used to transmit the signal to users [5]. To eliminate interference, each SC severs at most one user. A binary SC assignment variable, $x_{k, n}$, is introduced, and $x_{k, n}=1$ if SC $n$ is allocated to $k^{t h}$ user in the WIT phase and $x_{k, n}=0$ otherwise. The channel gain of the link from the AP to the $k^{t h}$ user on $n^{t h} \mathrm{SC}$ is denoted as $\mathbf{g}_{k, n} \in \mathbb{C}^{N_{T} \mathrm{x} 1}$. The transmit power of the AP assigned on SC $n$ for data transmission to the $k^{t h}$ user is denoted as $p_{k, n}$. Therefore, the achievable rate of $k^{t h}$ user on the $n^{t h}$ $\mathrm{SC}$ is given as $R_{k, n}=W\left(1-\tau_{0}\right) x_{k, n} \log \left(1+p_{k, n} \bar{u}_{k, n}\right)$, where $\bar{u}_{k, n}=\left\|\mathbf{g}_{k, n}\right\|^{2} /\left(W \delta^{2}\right)$ with $\delta^{2}$ being the noise spectral density. Then, the total sum-rate of the OFDMA-enabled WPCN scheme can be formulated as

$$
R_{\text {sum }}\left(\tau_{0}, \mathbf{p}, \mathbf{x}\right)=\sum_{k \in \mathcal{K}} \sum_{n \in \mathcal{N}} R_{k, n},
$$

where $\mathbf{p} \triangleq\left\{p_{k, n}, \forall k, \forall n\right\}, \mathbf{x} \triangleq\left\{x_{k, n}, \forall k, \forall n\right\}$.

\section{B. Power Consumption Model}

As in [6], the total energy consumption of the OFDMAenabled WPCN system includes two parts, the WET and WIT phases. During the WET phase, the energy consumption of the system is formulated as

$$
E_{W E T}\left(\tau_{0}, P_{0}\right)=P_{0} \tau_{0}-E_{\Theta} \tau_{0}+P_{c} \tau_{0},
$$

where

$$
E_{\Theta} \triangleq \eta P_{0} \sum_{n \in \mathcal{N}}\left\|\mathbf{h}_{p, n}\right\|^{2}
$$

and $P_{c}$ is the circuit power of the PS, $P_{0} \tau_{0}-E_{\Theta} \tau_{0}$ is the energy loss due to wireless channel propagation. During the WIT phase, the energy consumption at the AP is given by

$$
E_{W I T}\left(\tau_{0}, \mathbf{p}, \mathbf{x}\right)=\left(1-\tau_{0}\right)\left(\sum_{k \in \mathcal{K}} \sum_{n \in \mathcal{N}} x_{k, n} p_{k, n}+p^{c}\right),
$$

where $p^{c} \triangleq \sum_{k \in \mathcal{K}} p_{k, c}+P_{A, c}$ with $p_{k, c}$ and $P_{A, c}$ being the circuit powers of the $k^{\text {th }}$ user and the AP, respectively. Thus, the total energy consumption of the system is modeled as

$$
E_{\text {tot }}\left(\tau_{0}, \mathbf{p}, \mathbf{x}, P_{0}\right)=E_{W E T}\left(\tau_{0}, P_{0}\right)+E_{W I T}\left(\tau_{0}, \mathbf{p}, \mathbf{x}\right) .
$$

\section{Problem Formulation}

The system EE can be determined by the ratio of the sum-rate to the total consumed system energy, i.e., $\alpha_{D}=$ $\frac{R_{\mathrm{sum}}\left(\tau_{0}, \mathbf{p}, \mathbf{x}\right)}{E_{\mathrm{tot}}\left(\tau_{0}, \mathbf{p}, \mathbf{x}, P_{0}\right)}$ [6]. Therefore, the EE maximization problem can be mathematically formulated as

$$
\begin{aligned}
\max _{\substack{\tau_{0}, \mathbf{p}, \mathbf{x}, P_{0}}} \frac{\sum_{k \in \mathcal{K}} \sum_{n \in \mathcal{N}} W\left(1-\tau_{0}\right) x_{k, n} \log \left(1+p_{k, n} \bar{u}_{k, n}\right)}{E_{W E T}\left(\tau_{0}, P_{0}\right)+E_{W I T}\left(\tau_{0}, \mathbf{p}, \mathbf{x}\right)} \\
\text { s.t. C1: } P_{0} \leq P_{\max }, \quad \mathrm{C} 2: p_{k, n} \geq 0, \forall k, \forall n \\
\quad \mathrm{C} 3: x_{k, n} \in\{0,1\}, \forall k, \forall n, \mathrm{C} 4: \sum_{k \in \mathcal{K}} x_{k, n}=1, \forall n, \quad \text { (6) } \\
\quad \mathrm{C} 5:\left(1-\tau_{0}\right)\left(\sum_{k \in \mathcal{K}} \sum_{n \in \mathcal{N}} x_{k, n} p_{k, n}+P_{A, c}\right) \leq E_{\Theta} \tau_{0}, \forall k, \forall n \\
\quad \mathrm{C} 6: 0 \leq \tau_{0} \leq 1,
\end{aligned}
$$

where $E_{W E T}\left(\tau_{0}, P_{0}\right), E_{\Theta}$ and $E_{W I T}\left(\tau_{0}, \mathbf{p}, \mathbf{x}\right)$ are given as in (2), (3) and (4), respectively.

Our objective is to maximize the system EE subject to constraints on transmit power of the PS, subcarrier assignment and power allocation at the AP. C1 denotes the peak power $P_{\max }$ of the PS. C3 and C4 indicate that one SC is assigned to at most one link. C5 implies that the total energy consumed by the AP in the WIT phase should be less than the harvested energy in the WET phase. The problem in (6) is a non-convex and an MINLP problem. Note that the fractional form of the objective and the presence of constraint $\mathrm{C} 1$ related to the transmit power of PS make our problem different and more 
difficult to solve compared to the problem presented in [5]. Finding a solution to our problem becomes more troublesome than that of [5] due to the presence of optimized variables and the strong coupling among these variables in both the numerator and denominator. Thus, a direct application of the solution given in [5] will result in a poor permanence and thus, a simple but efficient method is required.

\section{SOLUTION TO ENERGY EFFICIENCY OF OFDMA-ENABLED WPCN}

We first determine the transmit power of the PS.

Proposition 1: The transmit power of the PS should be transmitted with a peak power $P_{\max }$ during the WET phase.

Proof: Please see Appendix.

Hence, to solve problem (6), we only need to determine the optimal $\tau_{0}, \mathbf{x}$ and $\mathbf{p}$. We can rewrite C5 as

$$
\tau_{0} \geq \bar{\tau}_{0} \triangleq \frac{\sum_{k \in \mathcal{K}} \sum_{n \in \mathcal{N}} x_{k, n} p_{k, n}+P_{A, c}}{\sum_{k \in \mathcal{K}} \sum_{n \in \mathcal{N}} x_{k, n} p_{k, n}+P_{A, c}+E_{\Theta}^{\max }},
$$

where $E_{\Theta}^{\max }$ is given in (3) with $P_{0}=P_{\max }$.

It is easy to verify that the objective function in (6) is nonincreasing with respect to $\tau_{0}$ by taking the first-order derivative and then checking the sign. The inequality in (7) should be active, i.e., $\tau_{0}=\bar{\tau}_{0}$.

Substituting $\bar{\tau}_{0}$ into (1) and (5), we obtain

$$
\begin{aligned}
\bar{R}_{\text {sum }}(\mathbf{p}, \mathbf{x})= & \frac{W E_{\Theta}^{\max } \sum_{k \in \mathcal{K}} \sum_{n \in \mathcal{N}} x_{k, n} \log \left(1+p_{k, n} \bar{u}_{k, n}\right)}{\sum_{k \in \mathcal{K}} \sum_{n \in \mathcal{N}} x_{k, n} p_{k, n}+P_{A, c}+E_{\Theta}^{\max }}, \\
\bar{E}_{\text {tot }}(\mathbf{p}, \mathbf{x})= & \left(\frac{\sum_{k \in \mathcal{K}} \sum_{n \in \mathcal{N}} x_{k, n} p_{k, n}+P_{A, c}}{\sum_{k \in \mathcal{K}} \sum_{n \in \mathcal{N}} x_{k, n} p_{k, n}+P_{A, c}+E_{\Theta}^{\max }}\right) \Gamma \\
& +\frac{E_{\Theta}^{\max }\left(\sum_{k \in \mathcal{K}} \sum_{n \in \mathcal{N}} x_{k, n} p_{k, n}+p^{c}\right)}{\sum_{k \in \mathcal{K}} \sum_{n \in \mathcal{N}} x_{k, n} p_{k, n}+P_{A, c}+E_{\Theta}^{\max }},
\end{aligned}
$$

respectively, where $\Gamma \triangleq P_{\max }-E_{\Theta}^{\max }+P_{c}$. Problem (6) can be rewritten as

$$
\begin{aligned}
& \max _{\mathbf{p}, \mathbf{x}} \frac{\bar{R}_{\text {sum }}(\mathbf{p}, \mathbf{x})}{\bar{E}_{\text {tot }}(\mathbf{p}, \mathbf{x})}=\frac{W \Omega_{0} \sum_{k \in \mathcal{K}} \sum_{n \in \mathcal{N}} x_{k, n} \log \left(1+p_{k, n} \bar{u}_{k, n}\right)}{\sum_{k \in \mathcal{K}} \sum_{n \in \mathcal{N}} x_{k, n} p_{k, n} \Omega_{1}+\Omega_{2}} \\
& \text { s.t. C2, C3, C4, }
\end{aligned}
$$

where $\Omega_{0} \triangleq E_{\Theta}^{\max }, \Omega_{1} \triangleq P_{\max }+P_{c}, \Omega_{2} \triangleq P_{A, c} \Gamma+\Omega_{0} p^{c}$.

Based on the fractional form of the objective function of the problem in (10), the Dinkelbach method is applied to address this issue. We first transform (10) into a subtractive form and then address the transformed problem. According to [6], the form of the EE maximization problem as

$$
\alpha_{D}^{*}=\max _{\mathbf{p}, \mathbf{x}}\left\{\bar{R}_{\text {sum }}(\mathbf{p}, \mathbf{x}) / \bar{E}_{\text {tot }}(\mathbf{p}, \mathbf{x})\right\}
$$

can be transformed into an equivalent problem in the subtractive form as follows:

$$
\begin{aligned}
& \max _{\mathbf{p}, \mathbf{x}}\left\{\bar{R}_{\text {sum }}(\mathbf{p}, \mathbf{x})-\alpha_{D}^{*} \bar{E}_{\text {tot }}(\mathbf{p}, \mathbf{x})\right\} \\
& \text { s.t. C2, C3, C4. }
\end{aligned}
$$

To obtain the maximum value $\alpha_{D}^{*}$, the iterative Dinkelbach method can be used by initializing a small $\alpha_{D}$ and then updating it during each iteration until convergence is achieved. Problem (10) can be rewritten as

$$
\begin{aligned}
& \max _{\mathbf{p}, \mathbf{x}} F(\mathbf{p}, \mathbf{x})=\bar{R}_{\text {sum }}(\mathbf{p}, \mathbf{x})-\alpha_{D} \bar{E}_{\text {tot }}(\mathbf{p}, \mathbf{x}) \\
& \text { s.t. C2, C3, C4. }
\end{aligned}
$$

In (13), $F(\mathbf{p}, \mathbf{x})$ can be simplified as

$$
F(\mathbf{p}, \mathbf{x})=\sum_{k \in \mathcal{K}} \sum_{n \in \mathcal{N}} x_{k, n} F_{k, n}\left(p_{k, n}\right)-\alpha_{D} \Omega_{2},
$$

where $F_{k, n}\left(p_{k, n}\right)=W \Omega_{0} \log \left(1+p_{k, n} \bar{u}_{k, n}\right)-\alpha_{D} \Omega_{1} p_{k, n}$.

To solve (13), we find the power allocation for given $\mathrm{SC}$ allocation $\mathbf{x}$ and $\alpha_{D}$. For given $\mathrm{SC}$ allocation $\mathbf{x}$ and $\alpha_{D}$, the power allocation over the SC with $x_{k, n}=1$ can be solved by addressing the following sub-problems: $\max _{p_{k, n}} F_{k, n}\left(p_{k, n}\right)$ [s.t.C2]. After solving $\frac{\partial F_{k, n}\left(p_{k, n}\right)}{\partial p_{k, n}}=$ $W \Omega_{0} \bar{u}_{k, n} /\left(1+p_{k, n} \bar{u}_{k, n}\right)-\alpha_{D} \Omega_{1}=0$, we have

$$
p_{k, n}^{*}=\left[W \Omega_{0} / \alpha_{D} \Omega_{1}-1 / \bar{u}_{k, n}\right]^{+},
$$

where $[x]^{+} \triangleq \max \{x, 0\}$. By substituting (15) into (14), we obtain $F(\mathbf{x})=\sum_{k \in \mathcal{K}} \sum_{n \in \mathcal{N}} x_{k, n} Q_{k, n}-\alpha_{D} \Omega_{2}$, where

$$
Q_{k, n}=W \Omega_{0} \log \left(1+p_{k, n}^{*} \bar{u}_{k, n}\right)-\alpha_{D} \Omega_{1} p_{k, n}^{*} .
$$

Thus, problem (13) becomes

$$
\max _{\mathbf{x}} F(\mathbf{x}) \quad[\text { s.t. C3, C4] . }
$$

It is easy to observe that the $Q_{k, n}$ is independent of the $x_{k, n}$ variable. Thus, the optimal $x_{k, n}^{*}$ for problem (17) is found as

$$
x_{k, n}^{*}= \begin{cases}1, & \text { if } k=k^{*} \\ 0, & \text { otherwise, }\end{cases}
$$

where $k^{*}=\underset{k}{\arg \max } Q_{k, n}$. In summary, a solution to the EE maximization problem in (10) is presented in Algorithm 1.

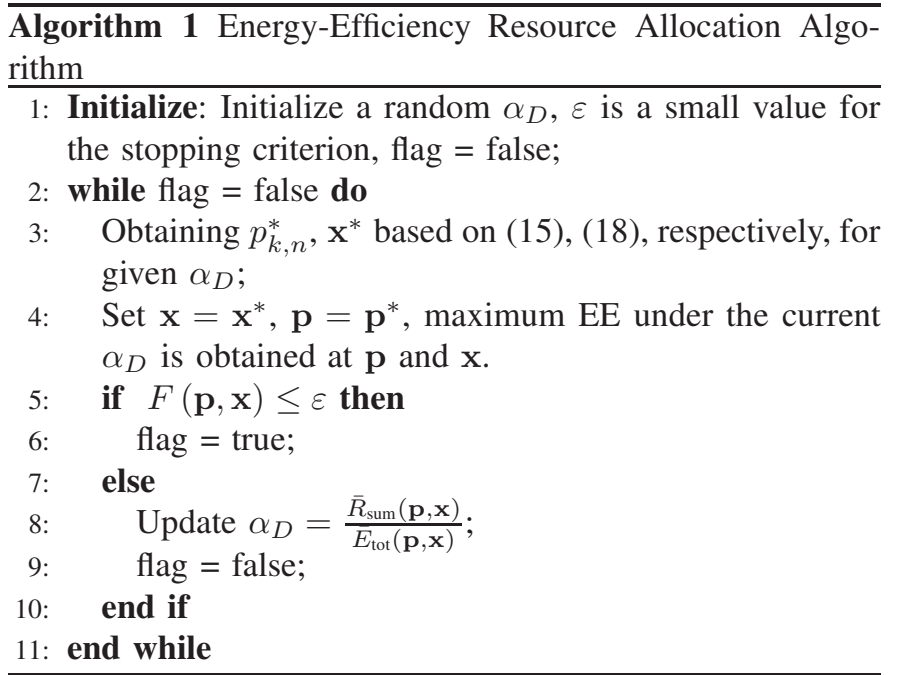

\section{NUMERICAL RESULTS}

In this section, we provide simulation results to demonstrate the effectiveness of the proposed resource allocation solution. It is assumed that channel gains are modeled as $10^{-3} \lambda d^{-\beta}$, 
where $\beta=2.4$ is the pathloss exponent and $\lambda$ is the exponential distribution Rayleigh with a unit mean. The positions of the PS and AP are located at $\{0 \mathrm{~m}, 0 \mathrm{~m}\}$ and $\{0 \mathrm{~m}, 10 \mathrm{~m}\}$, respectively. The positions of users are randomly distributed within the area of the square, assuming that the $\mathrm{x}$-coordinate positions are $\{-50 \mathrm{~m}, 50 \mathrm{~m}\}$ and $\mathrm{y}$-coordinate positions are $\{-50 \mathrm{~m}, 50 \mathrm{~m}\}$. Some other important simulation parameters are provided in Table I.

TABLE I

SIMULATION PARAMETERS

\begin{tabular}{|c|c|}
\hline Parameter & Value \\
\hline System bandwidth, $B$ & $5 \mathrm{MHz}$ \\
\hline Bandwidth for each SC, $W$ & $78 \mathrm{kHz}[13]$ \\
\hline Number of subcarriers, $N$ & 64 \\
\hline Noise spectral density, $\delta^{2}$ & $-174 \mathrm{dBm} / \mathrm{Hz}$ \\
\hline Energy conversion efficiency, $\eta$ & 0.8 \\
\hline Circuit power of the PS, $P_{c}$ & $5 \mathrm{dBm}$ \\
\hline Circuit power of the AP, $P_{A, c}$ & $5 \mathrm{dBm}$ \\
\hline Circuit power of each user, $p_{k, c}$ & $0 \mathrm{dBm}$ \\
\hline
\end{tabular}

In Table II, we provide the complexity analysis of the proposed scheme and other ones, i.e., equal power allocation (EPA), random SC allocation (RSA), fixed energy time (FET), and sum rate-based scheme [5]. The term by $\psi$ denotes the number of iterations needed to update the EE value $\alpha_{D}$ in Step 8 (i.e., outer loop) of Algorithm 1. The term by $\epsilon$ denotes a small value for the stopping criterion as presented in Algorithm 2 of [5].

TABLE II

COMPLEXITY ANALYSIS FOR DIFFERENT SCHEMES

\begin{tabular}{|c|c|}
\hline Scheme & Complexity \\
\hline Proposed scheme & $\mathcal{O}(\psi(N+N K))$ \\
\hline RSA scheme & $\mathcal{O}(\psi N K)$ \\
\hline EPA scheme & $\mathcal{O}(\psi N)$ \\
\hline Sumrate-based scheme & $\mathcal{O}\left(\log _{2}(1 / \epsilon)(N+N K)\right)[5]$ \\
\hline FET scheme & $\mathcal{O}(\psi(N+N K))$ \\
\hline
\end{tabular}

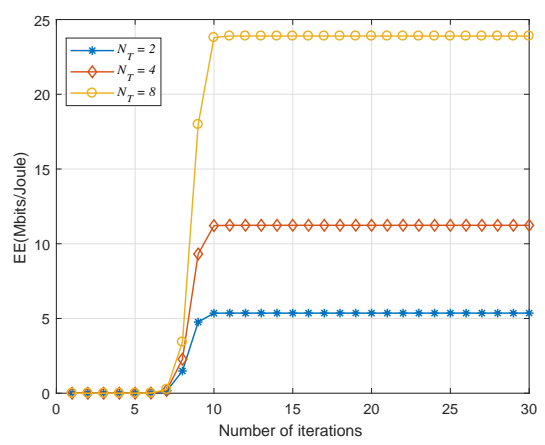

Fig. 1. Convergence of the proposed algorithm with different number of antennas at the AP, $P_{\max }=45 \mathrm{dBm}, K=10$.

Fig. 1 shows the convergence evolution of our proposed algorithm under different numbers of antennas at the AP with
$K=10$ and $P_{\max }=45 \mathrm{dBm}$. It can be seen that the algorithm converges after approximately 10 iterations. The figure also reveals that the system EE increases as the number of antennas at the AP increases, due to the higher amount of harvested energy at the AP.

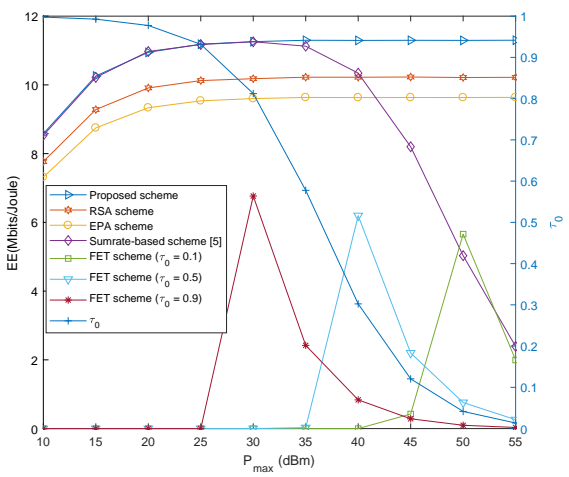

Fig. 2. Energy efficiency for all schemes and EH durations $\tau_{0}$ of the proposed scheme versus PS's transmit power, $K=10, N_{T}=4$.

In Fig. 2, the system EE of our proposed scheme and several schemes, including the EPA scheme, the RSA and the FET $\left(\tau_{0}=0.1,0.5,0.9\right)$, the sumrate-based scheme, are shown as functions of the transmit power of the PS. From the figure, for the proposed scheme, the EE gradually increases as the maximum transmit power of the PS increases, and becomes saturated when the PS has a sufficient maximum transmit power. The same phenomenon is observed for the EPA and RSA schemes. However, the performance of the two baseline schemes is lower than that of the proposed scheme due to the lack of SC assignment for RSA and power allocation for EPA. Furthermore, the performance of the sumrate-based scheme is the same as that of the proposed scheme but better than that of the RSA, EPA, and FET schemes with a small $P_{\max }$. However, the EE of the based-sumrate scheme starts to decreases when $P_{\max }$ is sufficiently large. This is because there is a trade-off between the system throughput and the energy consumption resulting in saturation of the system EE for the proposed scheme with large $P_{\max }$. Meanwhile, for the based-sumrate scheme, with the same large $P_{\max }$, an increase in energy consumption occurs faster than an increase in the throughput leading to a reduction in the system EE.

For the FET schemes, the EE is initially in an inactive state and then significantly decreases after reaching its maximum value. This is because initially, a small $P_{\max }$ requires a longer $\mathrm{EH}$ duration; therefore, because of a fixed EH time, the system has insufficient EH time for the WET phase. A larger $P_{\max }$ reduces the $\mathrm{EH}$ time, which results in a decrease in the circuit energy consumption, which increases the time for the WIT phase of the proposed algorithm-based scheme. However, due to a fixed EH time, the FET-based schemes are not able to take advantage of the high $P_{\max }$ and may not have sufficient time for the WIT phase to improve EE, whereas the circuit energy consumption for the WET phase increases. As a result, the EE of the FET schemes significantly decreases after reaching the maximum value. This further confirms the effectiveness of the 
proposed solution.

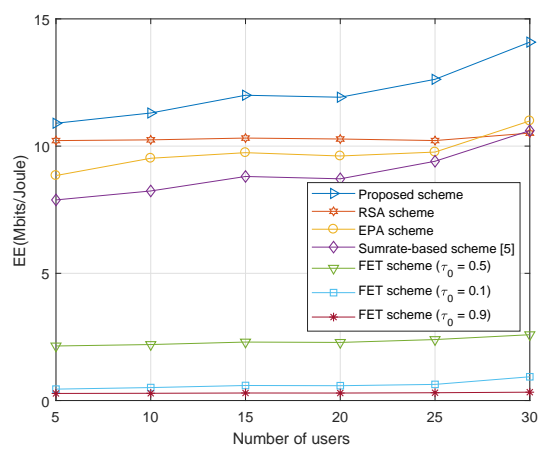

Fig. 3. Energy efficiency versus number of users, $P_{\max }=45 \mathrm{dBm}, N_{T}=4$.

In Fig. 3, we evaluate the system EE as the number of users is varied, where $P_{\max }=45 \mathrm{dBm}$ and $N_{T}=4$. We can see that there is a slight increase in the system EE for both schemes as the number of users increases. This is because a larger number of users leads to a higher multiuser diversity gain, which in turn results in a higher throughput of the system. The increase in the total system power consumption caused by an increase in the number of users is insignificant. Moreover, it is observed that our proposed solution outperforms the other schemes.

\section{CONCLUSION}

We have studied the problem of maximizing the EE of the OFDMA-enabled WPCN. The MINLP problem is efficiently solved by the iterative based Dinkelbach method. The novelty of the proposed algorithm is attributed to the fact that in each iteration closed-form solutions are found. The desirability of jointly optimizing $\mathrm{EH}$ time, SC assignment, and power allocation is justified by simulation results.

\section{APPENDIX}

\section{PROOF OF PROPOSITION 1}

From (C5) in (6) and (3), we have

$$
P_{0} \geq \bar{P}_{0} \triangleq \frac{\left(1-\tau_{0}\right)\left(\sum_{k \in \mathcal{K}} \sum_{n \in \mathcal{N}} x_{k, n} p_{k, n}+P_{A, c}\right)}{\tau_{0} \eta \sum_{n \in \mathcal{N}}\left\|\mathbf{h}_{p, n}\right\|^{2}} \text {. }
$$

We observe that the objective function of problem (6) is nonincreasing with respect to $P_{0}$. It is easy to verify for the first order derivative of the objective function. Thus, from (C5), the optimal $P_{0}$ should hold for given power allocation

$$
P_{0}=\bar{P}_{0} .
$$

Substituting (20) into (5), the total energy consumption can be expressed as

$$
\tilde{E}_{\text {tot }}\left(\mathbf{p}, \mathbf{x}, \tau_{0}\right)=\left(1-\tau_{0}\right) \frac{\Psi_{0} \Psi_{1}}{\Psi_{2}}+\tau_{0} P_{c}+\left(1-\tau_{0}\right) \Psi_{3},
$$

where $\Psi_{0} \triangleq \sum_{k \in \mathcal{K}} \sum_{n \in \mathcal{N}} x_{k, n} p_{k, n}+P_{A, c}, \quad \Psi_{1} \triangleq$ $1-\eta \sum_{n \in \mathcal{N}}\left\|\mathbf{h}_{p, n}\right\|^{2}, \quad \Psi_{2} \triangleq \eta \sum_{n \in \mathcal{N}}\left\|\mathbf{h}_{p, n}\right\|^{2}, \quad \Psi_{3} \triangleq$ $\sum_{k \in \mathcal{K}} \sum_{n \in \mathcal{N}} x_{k, n} p_{k, n}+p^{c}$.
Substituting (1) and (21) into (6), the EE maximization problem becomes

$$
\begin{gathered}
\max _{\tau_{0}, \mathbf{p}, \mathbf{x}} \Phi\left(\mathbf{p}, \mathbf{x}, \tau_{0}\right)=R_{\text {sum }}\left(\mathbf{p}, \mathbf{x}, \tau_{0}\right) / \tilde{E}_{\text {tot }}\left(\mathbf{p}, \mathbf{x}, \tau_{0}\right) \\
\text { s.t. } \overline{\mathrm{C}} 1:\left(1-\tau_{0}\right) \Psi_{0} /\left(\tau_{0} \Psi_{2}\right) \leq P_{\max }, \\
\text { C2 }, \mathrm{C} 3, \mathrm{C} 4, \mathrm{C} 6 .
\end{gathered}
$$

From $\overline{\mathrm{C}} 1$, we have $\tau_{0} \geq \Psi_{0} /\left(\Psi_{0}+\Psi_{2} P_{\max }\right)$. The first derivative of the objective of (22) is given as

$$
\begin{aligned}
\frac{\partial \Phi}{\partial \tau_{0}}= & -\frac{\sum_{k \in \mathcal{K}} \sum_{n \in \mathcal{N}} W x_{k, n} \log \left(1+p_{k, n} \bar{u}_{k, n}\right) \tau_{0} P_{c}}{\left(\tilde{E}_{\text {tot }}\left(\mathbf{p}, \mathbf{x}, \tau_{0}\right)\right)^{2}} \\
& -\frac{\sum_{k \in \mathcal{K}} \sum_{n \in \mathcal{N}} W\left(1-\tau_{0}\right) x_{k, n} \log \left(1+p_{k, n} \bar{u}_{k, n}\right) P_{c}}{\left(\tilde{E}_{\text {tot }}\left(\mathbf{p}, \mathbf{x}, \tau_{0}\right)\right)^{2}} .
\end{aligned}
$$

The objective function of problem (22) is a non-increasing function with respect to $\tau_{0}$. Thus, from $\overline{\mathrm{C}} 1$, the optimal $\tau_{0}$ should be hold $\tau_{0}=\Psi_{0} /\left(\Psi_{0}+\Psi_{2} P_{\max }\right)$. Substituting $\tau_{0}$ into (20), we have

$$
P_{0}=\frac{\left(\Psi_{2} P_{\max } /\left(\Psi_{0}+\Psi_{2} P_{\max }\right)\right) \Psi_{0}}{\left(\Psi_{0} /\left(\Psi_{0}+\Psi_{2} P_{\max }\right)\right) \Psi_{2}}=P_{\max } .
$$

\section{REFERENCES}

[1] K. Ali, H. X. Nguyen, Q. Vien, P. Shah, and Z. Chu, "Disaster Management Using D2D Communication With Power Transfer and Clustering Techniques," IEEE Access, vol. 6, pp. 14643-14654, Jan. 2018.

[2] Q. Pham, F. Fang, V. N. Ha, M. J. Piran, M. Le, L. B. Le, W. Hwang, and Z. Ding, "A Survey of Multi-Access Edge Computing in 5G and Beyond: Fundamentals, Technology Integration, and State-of-the-Art," IEEE Access, vol. 8, pp. 116974-117 017, 2020.

[3] T. Do, V. Nguyen, O. Shin, and B. An, "Simultaneous Uplink and Downlink Transmissions for Wireless Powered Communication Networks," IEEE Communications Letters, vol. 23, no. 2, pp. 374-377, Feb. 2019.

[4] C. Lee, "Wireless Information and Power Transfer for Communication Recovery in Disaster Areas," in Proceeding of IEEE International Symposium on a World of Wireless, Mobile and Multimedia Networks 2014, Sydney, NSW, Australia, Jun. 2014, pp. 1-4.

[5] T. Nguyen, V. Nguyen, J. Lee, and Y. Kim, "Sum Rate Maximization for Multi-User Wireless Powered IoT Network with Non-Linear Energy Harvester: Time and Power Allocation," IEEE Access, vol. 7, pp. 149 698-149710, 2019.

[6] Q. Wu, W. Chen, and J. Li, "Wireless Powered Communications With Initial Energy: QoS Guaranteed Energy-Efficient Resource Allocation," IEEE Communications Letters, vol. 19, no. 12, pp. 2278-2281, Dec. 2015.

[7] X. Lin, L. Huang, C. Guo, P. Zhang, M. Huang, and J. Zhang, "EnergyEfficient Resource Allocation in TDMS-Based Wireless Powered Communication Networks," IEEE Communications Letters, vol. 21, no. 4, pp. 861-864, Apr. 2017.

[8] M. Song and M. Zheng, "Energy Efficiency Optimization For Wireless Powered Sensor Networks With Nonorthogonal Multiple Access," IEEE Sensors Letters, vol. 2, no. 1, pp. 1-4, Mar. 2018.

[9] Q. Wu, G. Y. Li, W. Chen, and D. W. K. Ng, "Energy-Efficient Small Cell With Spectrum-Power Trading," IEEE Journal on Selected Areas in Communications, vol. 34, no. 12, pp. 3394-3408, Dec 2016.

[10] O. Amjad, E. Bedeer, and S. Ikki, "Energy-Efficiency Maximization of Self-Sustained Wireless Body Area Sensor Networks," IEEE Sensors Letters, vol. 3, no. 12, pp. 1-4, Dec 2019.

[11] F. Yang, W. Xu, Z. Zhang, L. Guo, and J. Lin, "Energy Efficiency Maximization for Relay-Assisted WPCN: Joint Time Duration and Power Allocation," IEEE Access, vol. 6, pp. 78 297-78 307, 2018.

[12] Y.-E. Wang, X. Lin, A. Adhikary, A. Grovlen, Y. Sui, Y. Blankenship, J. Bergman, and H. S. Razaghi, "A Primer on 3GPP Narrowband Internet of Things," IEEE Communications Magazine, vol. 55, no. 3, pp. 117123, Mar. 2017.

[13] Y. Sun, D. W. K. Ng, Z. Ding, and R. Schober, "Optimal Joint Power and Subcarrier Allocation for Full-Duplex Multicarrier Non-Orthogonal Multiple Access Systems," IEEE Transactions on Communications, vol. 65, no. 3, pp. 1077-1091, Mar. 2017 